\title{
Subject Index, Vol. 76, 1997
}

This index does not contain items of the report of the Chromosome 15 Mapping Workshop which is published in this volume.

Animals

Blarina carolinensis (shrew) 153

bovine 36

chicken 229

chimpanzee 189

Chinese hamster 58,128

finch 77

Monodelphis domestica (opossum) 115

mouse $164,179,180,185$

Muntiacus crinifrons (black muntjac) 159

rat 47,101

swine 27, 53, 109

Banded chromosome analysis bovine(G) 36 chicken(DAPI) 229 chimpanzee(DAPI) 189

Chinese hamster(D API) 128 Chinese hamster(DAPI-Q, C) 58 human(C/AluI) 94

human(DAPI) 19, 45, 139, 172, 181, 189 human(DAPVAD) 72, 176 human(G) 23, 123, 172 , 196 human(GTG) 68, 167 human(NOR) 172 human(R) 17,34,87,172, 183 mouse(R) 179, 180, 185 muntjac(DAPI) $159 \operatorname{opossum}(\mathrm{G}) 115 \operatorname{rat}(\mathrm{Q}) 47 \operatorname{shrew}(\mathrm{G}) 153 \operatorname{swine}(\mathrm{G}) 109$ swine(RBP) 53

Cell cycle

cell growth inhibitor 176

induction of anaphase/telophase abnormalities 61 Chromosome aberration

abberations induced by topoisomerase inhibition 61

aneuploidy $61,134,192$

deletions 202,214

gene amplification 128

insertions 14, 23, 72

isochromosome 36,196

nondisjunction 61,192

pericentric inversions 189

rearrangements 159

rearrangements in AZT treated cells 128

rearrangements induced by azacytidine 196

Robertsonian rearrangements 153

translocations 68

trisomy $1 \mathrm{lq}$ and monosomy $5 \mathrm{q} 68$

whole arm deletions 196 Chromosome mapping workshops

chromosome 151 Chromosome preparation 
chromosome stretching 167 Comparative gene mapping

chimpanzee 189

rat 47

swine 27, 53, 109

Evolution

5 sRNA repeats 101

black muntj ac karyotype 159

Chinese hamster marker chromosome 58

human chromosome 18

LCAT gene cluster 53

shrew karyotype 153

Flow cytometry

black muntjac 159 Fluorescent in situ hybridization (FISH) and fluorescent BrdU

incorporation 128 and pulsed field gel electrophoreisis 208 breakpoint determination 23

chromosome specific painting probes 23,27 ,

$58,68,72$ comparative chromosome painting 53,159 ,

189 comparative genomic hybridization 68 deletion analysis 202 direct $\mathrm{R}$ banding 87 fiber-

FISH 20

interphase FISH 49, 94, 115, 134, 208 multicolor FISH 23, 45, 49, 58, 61, 134, 159,

189,192,202,208 restriction endonuclease digestion 94

Gene mapping bovine

SRY 36 chicken

MAX 229 human

COPA 139

CRABP1, CRABP2 17

CST6 45

ERBB2 34

FGF13 183

GDIA1 172

HLCS 179

IFNGR 1181

IHH 187

ING1 176

KPNA3 87

POMC 221

PRKY, PRKXP1, PRKXP2 49

PTK7 43

skeletal muscle ESTs 144

SLC6A10 19

TAF2H 41

THRSP 219

TNA 39

TRIO 107 mouse

Extl 164

Fgfl2 185

Hies 179 
Slc Пal 180 opossum

6PGD, G6PD, GPI, GOT1, HPRT, LDHA, LDHB, PGK, TK 115 rat

Rn5s, Rn5sp 101

Tbxas 47

swme

AHC, AMH, CYP19, FTZF1, SOX2, SOX9.WT1 109 Genetic damage

AZT induced rearrangements 128 multilocus loss of heterozygosity 214 single base insertion 14

Heterochromatin

methylation in sperm 123

rearrangements induced by methylation inhibitors 196 Human disorders

alkaptonuria 14

breast cancer 34,45

Burkitt's lymphoma, melanoma 181

colon carcinoma 43

ESS1 (self healing squamous epitheliomata) 208

gastric cancer and head and neck squamous carcinoma 176

Hodgkin's disease 134

hypophosphatemia 180

ICF syndrome 123, 196

immunodeficiency and facial dysmorphism 123

intersexuality 109

lipid disorders 219

multiple exostoses formation 164

pleomorphic adenoma 23

X-ray sensitivity 87 Hybrids

1 lpl5 radiation hybrid panel 41

chromosome 3 hybrid panel 39

human-chicken monochromosome panel 72

human-rodent 43, 49, 144, 223

opossum-eutherian 115

pig-rodent 109

radiation hybrid 187,223

Xpl 1 hybrid panel 223

Immunofluorescence

protein localization 176 Interspecific backcross mapping

mouse 164

Karyotype

black muntjac (Muntiacus crinifrons)

159 in vitro karyotype evolution 58 opossum (Monodelphis domestica) 115 shrew (Blarina carolinensis) 153

Linkage

chicken linkage group 7229 human 3 p22 $\rightarrow$ p21 39 mouse chromosome 15164 swine

chromosome 627,53

Meiosis

aneuploidy in sperm 134,192 
male muntjac 159

methylation of sperm chromosomes 123

nondisjunction 192

\section{KAIUiER}

(C) 1997 S. KargerAG, Basel

235

E-Mail karger@karger.ch Fax+4161306 1234 http://www. karger. ch

Sequence

Radioactive in situ hybridization (RISH) human COPA 139

human FGF13 183 human HGD 14

Microdissection and microcloning swine chromosome 627

Nucleolus

sex-specific size in neuronal tissue 77

Physical mapping

$8 \mathrm{q} 12$ breakpoint 23

alphoid and rDNA 20

distal $17 \mathrm{q}$ hybrid panel 172

human9q22.3 208

human skeletal muscle ESTs 144

human Xp 11223

sex reversal genes in pig 109

swine chromosome 653 PRINS

disomy in sperm 192

mouse Fgj 12185

pigAMH, CYP19, FTZFl, WT1 109 rat5sRNA 101 Repeats

AM digestion of alphoid and classical

satellite DNA 94 amplification induced by AZT 128 interstitial telomeric sequences 58

polymorphic microsatellite markers to

identify LOH 214 rat5sRNA 101 rDNA repeats as size markers on extended

fibers 20 swine chromosome 6 microsatellite markers

27 tandem repeats at Xp 11223

human KPNA3 87 mRNA initiation site 139 m urine E¹/8/ 164 PRKY, PRKXP1, PRKXP2 49

rat $R n 5 s, R n 5 s p 101$ Sex chromosomes

bovine XY female 36 XÄY1Y2Y3 system 159

Techniques

digital image analysis of FISH signals 94

dual-color PRINS 192

gene insertion-type targeted integration 72

metaphase spreading 167

sequence-independent FISH probe amplification 202

targeted gene walking with low stringency PCR 139

236

Cytogenet Cell Genet Vol. 76, 1997 